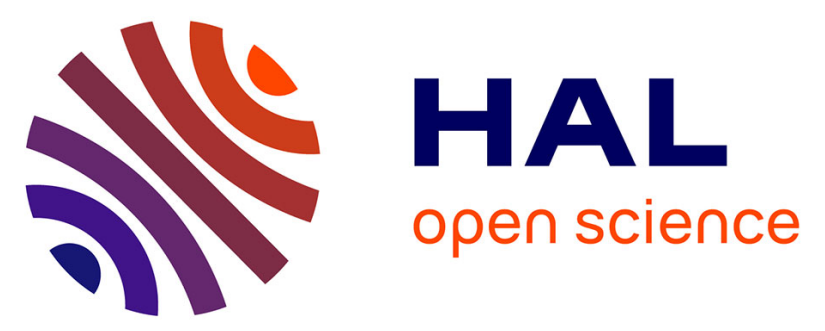

\title{
Event related potentials elicited by violations of auditory regularities in patients with impaired consciousness.
}

Frédéric Faugeras, Benjamin Rohaut, Nicolas Weiss, Tristan Bekinschtein, Damien Galanaud, Louis Puybasset, Francis Bolgert, Claire Sergent, Laurent Cohen, Stanislas Dehaene, et al.

\section{To cite this version:}

Frédéric Faugeras, Benjamin Rohaut, Nicolas Weiss, Tristan Bekinschtein, Damien Galanaud, et al.. Event related potentials elicited by violations of auditory regularities in patients with impaired consciousness.. Neuropsychologia, 2012, 50 (3), pp.403-18. 10.1016/j.neuropsychologia.2011.12.015 . hal-00719338

\section{HAL Id: hal-00719338 \\ https://hal.science/hal-00719338}

Submitted on 19 Jul 2012

HAL is a multi-disciplinary open access archive for the deposit and dissemination of scientific research documents, whether they are published or not. The documents may come from teaching and research institutions in France or abroad, or from public or private research centers.
L'archive ouverte pluridisciplinaire HAL, est destinée au dépôt et à la diffusion de documents scientifiques de niveau recherche, publiés ou non, émanant des établissements d'enseignement et de recherche français ou étrangers, des laboratoires publics ou privés. 


\section{Event related potentials elicited by violations of auditory regularities in patients with impaired consciousness}

\section{Authors :}

Frédéric Faugeras ${ }^{1,5,9}$, Benjamin Rohaut ${ }^{1,5,9}$, Nicolas Weiss ${ }^{2,}$, Tristan Bekinschtein $^{7}$, Damien Galanaud ${ }^{4,5,9}$, Louis Puybasset ${ }^{3,8}$, Francis Bolgert ${ }^{2}$, Claire Sergent $^{5,9}$, Laurent Cohen ${ }^{2,5,8,9}$, Stanislas Dehaene ${ }^{6}$, Lionel Naccache ${ }^{1,2,5,8,9^{*}}$

(1) AP-HP, Groupe hospitalier Pitié-Salpêtrière, Department of Neurophysiology, Paris, France

(2) AP-HP, Groupe hospitalier Pitié-Salpêtrière, Department of Neurology, Paris, France

(3) AP-HP, Groupe hospitalier Pitié-Salpêtrière, Department of Neurosurgical Intensive Care Unit, Paris, France

(4) AP-HP, Groupe hospitalier Pitié-Salpêtrière, Department of Neuroradiology, Paris, France

(5) INSERM, ICM Research Center, UMRS 975, Paris, France

(6) INSERM-CEA Cognitive Neuroimaging unit |CEA/SAC/DSV/DRM/Neurospin center, Gif/Yvette cedex, France

(7) MRC-Cognition and Brain Sciences Unit, Cambridge, UK

(8) University Paris 6, Faculté de Médecine Pitié-Salpêtrière, Paris, France

(9) Institut du Cerveau et de la Moëlle épinière, Paris, France

*Corresponding Author

lionel.naccache@psl.aphp.fr 


\begin{abstract}
Improving our ability to detect conscious processing in non communicating patients remains a major goal of clinical cognitive neurosciences. In this perspective, several functional brain imaging tools are currently under development. Bedside cognitive event-related potentials (ERPs) derived from the EEG signal are a good candidate to explore consciousness in these patients because: 1) they have an optimal time resolution within the millisecond range able to monitor the stream of consciousness, 2) they are fully non-invasive and relatively cheap, 3) they can be recorded continuously on dedicated individual systems to monitor consciousness and to communicate with patients, 4) and they can be used to enrich patients' autonomy through brain-computer interfaces. We recently designed an original auditory rule extraction ERP test that evaluates cerebral responses to violations of temporal regularities that are either local in time, or global across several seconds. Local violations led to an early response in auditory cortex, independent of attention or the presence of a concurrent visual task, while global violations led to a late and spatially distributed response that was only present when subjects were attentive and aware of the violations. In the present work, we report the results of this test in 65 successive recordings obtained at bedside from 49 non-communicating patients affected with various acute or chronic neurological disorders. At the individual level, we confirm the high specificity of the 'global effect': only conscious patients presented this proposed neural signature of conscious processing. Here, we also describe in details the respective neural responses elicited by violations of local and global auditory regularities, and we report two additional ERP effects related to stimuli expectancy and to task learning, and we discuss their relations to consciousness.
\end{abstract}

KEY-WORDS: CONSCIOUSNESS / AUDITION / ERP / PATIENTS / VEGETATIVE STATE 


\section{Introduction}

"Is he/she conscious?" Far from being a purely philosophical abyssal issue, this question is a daily interrogation for the caregivers and health professionals of acute or chronic noncommunicating patients. Answers to this question are crucial to optimize the medical management of those patients, to specify the amount of efforts devoted to communicate with them, and to provide robust objective landmarks to the caregivers and close relatives of the patients in these extremely difficult situations where irrepressible emotions, subjective feelings and interpretative beliefs may be misleading and insufficient to guide medical strategy.

For many years, clinical examination and behavioural observation constituted the single approach to diagnose consciousness (Plum \& Posner, 1972). Consciousness is clinically defined in relation to the diverse neurological conditions where it is impaired or absent. Major principles can be derived from clinical neurology:

\subsection{Clinical markers of consciousness}

Consciousness requires wakefulness: the case of comatose states

First, a necessary but insufficient physiological condition to consciousness is wakefulness, that is to say the presence of waking periods during which the patient keeps his eyes open independently of external stimulations. Wakefulness is impaired in comatose states, in general anesthesia or in deep sleep stages in which patients are not conscious (Laureys, Owen, \& Schiff, 2004). The neural bases of wakefulness mostly involve complex brainstem and thalamic networks often regouped under the generic term of ascending reticular activating system (ARAS), (see (Moruzzi \& Magoun, 1949), and (Parvizi \& Damasio, 2001) for a recent review).

Consciousness is not wakefulness: the case of vegetative states

A more subtle alteration of consciousness is the vegetative state (VS), which is characterized by preserved wakefulness ((Jennett \& Plum, 1972), - even if circadian rhythms may not be strictly normal (Bekinschtein, Golombek, Simonetta, Coleman, \& Manes, 2009) -, in the absence of any purposeful behavior and of any sign of intentional reactions to the external environment. Note that VS is, by definition, a clinical syndrome and not a specific condition. For this reason, and in order to avoid too radical interpretations of patient's cognitive state 
only based on behavioural observations, a group of experts recently proposed the 'Unresponsive Wakefulness Syndrome' expression to describe VS (Laureys et al., 2010).

The mere existence of VS demonstrates that wakefulness and consciousness can be dissociated, and therefore that they cannot be identified one with another (Bernat, 2006). While VS can have a highly variable duration, from several days to a whole lifetime, other neurological situations can be described as 'transient VS': during complex partial epileptic seizures or during "petit mal absence" seizures for instance, a comparable dissociation between consciousness and wakefulness occurs, but on a much shorter time-scale, usually from a few seconds to several minutes (Blumenfeld \& Taylor, 2003).

\section{Transitions between VS and consciousness: the case of minimally conscious states (MCS)}

Neurological observations revealed that many patients presented fluctuating states which could be identified neither as VS or conscious states. These transitional states have recently been regrouped under the concept of minimally conscious states (MCS, (Giacino et al., 2002)). The behavioural distinction between VS and MCS requires an expertise in clinical assessment and can be based on the use of a dedicated scale: the revised version of the Coma Recovery Scale (CRS-R, see (Kalmar \& Giacino, 2005), adapted in many languages including French (Schnakers, Majerus et al., 2008)). For instance, while VS patients can show fast and transient saccadic responses to moving visual targets, the presence of sustained and reproducible visual pursuit is an index of MCS. Note that a recent work showed that the use of EMG signal in active motor paradigms is more sensitive than mere clinical examination of overt movements (Bekinschtein, Coleman, Niklison, Pickard, \& Manes, 2008).

\section{Motor neurological examination is a prerequisite: pitfalls of locked-in syndromes}

Prior to consciousness assessment, a detailed clinical checking of the functionality of motor pathways is absolutely necessary, as demonstrated by various clinical conditions in which a paralyzed but conscious patient can be misclassified as unconscious. "Locked in syndrome" usually secondary to brainstem strokes in the paramedian protuberance (Laureys et al., 2005), but also related conditions such as severe Guillain-Barré polyradiculoneuritis or severe amyotrophic lateral sclerosis are typical illustrations of this point.

\section{Covert cognitive impairments may underestimate consciousness}


Note also that the presence of massive cognitive impairments may be difficult to detect and may lead to an underestimation of consciousness. For instance, a non-communicating patient affected by a global aphasia (e.g.: massive left hemispheric lesion) will probably not demonstrate any adapted behavior even to basic verbal instructions. Similarly, massive impairments in anterograde memory, in working memory or executive functions can lead to an underestimation of the consciousness status.

In the light of these fundamental neurological principles, it is clear that purely behavioral observations have limited sensitivity, and only constitute indirect evidence of conscious processes. In some cases, the categorization of a patient as vegetative or minimally conscious is far from obvious. Thus, in many daily clinical situations, the inaugural question of this article is left unanswered: "Is he/she conscious?"

\subsection{Markers of consciousness derived from cognitive neuroscience}

A complementary approach to clinical neurology originates from cognitive neurosciences of consciousness. Although the issue remains debated, two decades of experimental and theoretical works have led to the characterization of psychological and neurophysiological attributes that may be unique to conscious processing (Seth, Dienes, Cleeremans, Overgaard, \& Pessoa, 2008). Many cognitive processes may occur unconsciously both in conscious subjects (Dehaene, Changeux, Naccache, Sackur, \& Sergent, 2006 ; Kouider \& Dehaene, 2007), in visual neglect patients or related patients (Driver \& Mattingley, 1998 ; Naccache, 2008), and in non conscious patients (Laureys, 2005 ; Owen et al., 2005), reaching such complex levels as abstract semantics, phonological or emotional processing. Still, three properties seem to be exclusively associated with conscious processing of reportable mental contents (Dehaene \& Naccache, 2001): (i) active maintenance of mental representations in working memory; (ii) strategical processing; and (iii) spontaneous intentional behavior (8). Similarly, while unconscious processing may engage multiple isolated cortical areas, neural signatures of conscious processing are defined by late and long-lasting brain activations that mobilize long-distance coherent thalamo-cortical networks, particularly involving bilateral prefrontal, cingulate and parietal areas (Dehaene, Changeux, Naccache, Sackur, \& Sergent, 2006 ; Gaillard et al., 2009).

On the basis of these studies, original experimental paradigms can therefore be designed in order to improve our ability to diagnose consciousness in non-communicating patients, beyond clinical evaluations. For instance, at the behavioral level, Bekinschtein and colleagues 
(Bekinschtein, Shalom et al., 2009) capitalized on the working memory property mentioned above, and used an eyeblink conditioning paradigm in which a tone stimulus can be paired with an air-puff delivered on the cornea. Delay conditioning, - where the conditioned stimulus and the unconditioned air-puff overlap in time - does not require conscious processing of the stimuli. In contrast, trace conditioning where a temporal gap is inserted between the two stimuli seems to require conscious processing in working memory (Clark \& Squire, 1998). Interestingly, they showed that some clinically defined VS patients were able to demonstrate both conditioning and trace conditionings. Functional brain-imaging approaches are also emerging (Coleman et al., 2009). For instance, Owen, Laureys and their colleagues (2006) probed with fMRI the active maintenance of task-instructed cognitive tasks, such the ability to perform motor or spatial imagery tasks for a extended duration of 30 seconds. Using this approach on 54 patients, they could identify 5 patients able to willfully modulate their brain activity (Monti et al., 2010). Among these 5 patients, two were clinically classified as VS. In one clinically MCS patient, fMRI could be used to define an arbitrary code and communicate a single bit of information (a yes/no answer), while such a communication was not possible behaviorally.

In parallel to such fMRI experiments, EEG paradigms may constitute a highly promising research direction for at least two reasons. First, EEG is a time-resolved tool able to sample brain activity at the millisecond scale. This offers a unique opportunity to monitor the flow of consciousness and eventually to interact with the patient in real-time. Second, given that EEG is a non-invasive technique, has a relatively low-cost and can be recorded at bedside, one may ultimately design dedicated systems for recurrent and even continuous daily recording of brain activity in patients. In that respect, EEG monitoring seems more likely to truthfully reflect VS and MCS patients' complex fluctuating states than a single fMRI scan lasting a few tens of minutes. Schnakers and her colleagues showed the utility of using active EEG paradigms to probe voluntary brain responses to stimuli. They could confirm the presence of conscious processing in a locked-in syndrome patient (Schnakers, Perrin et al., 2009), and in clinically defined MCS patients (Schnakers, Perrin et al., 2008).

\section{The 'local global' test of consciousness}

We recently designed an auditory paradigm that evaluates the cerebral responses to violations of temporal regularities (Bekinschtein, Dehaene et al., 2009). Local violations due to the unexpected occurrence of a single deviant sound amongst a repeated train of standard sounds led to an early response in auditory cortex, the mismatch negativity (MMN) ERP component, 
independent of attention and of the presence of a concurrent visual task. On the other hand, global violations, defined as the presentation of a rare and unexpected series of five sounds, led to a late and spatially distributed response that was only present when subjects were attentive and aware of the violations (P3b ERP component). We could detect the global effect in individual subjects using functional MRI and both scalp and intracerebral event-related potentials. The original publication (Bekinschtein, Dehaene et al., 2009) reported the results from 8 non communicating patients with disorders of consciousness (4 MCS and 4 VS) and confirmed that only conscious individuals presented a global effect (3 MCS patients). In a more recent work focusing on a larger sample of clinically defined VS patients, we confirmed the absence of global effect in the vast majority of patients, and identified 2 patients showing this neural signature of consciousness (Faugeras et al., 2011). Interestingly, these 2 patients showed unequivocal clinical signs of consciousness within the 3-4 days following ERP recording, strongly suggesting they were misclassified as VS due to limitations of clinical examination. Taken together, these observations were highly suggestive that the global effect might be a signature of conscious processing, although it can be absent in conscious subjects who are not aware of the global auditory regularities.

\subsection{Objectives of the present study}

In the present work, we prospectively explored the first 100 consecutive recordings obtained in 65 non-communicating patients (November 2008 to February 2010) with the 'local global' paradigm while recording their EEG activity with a high-density EEG system (see Figure 1), subsequently to a detailed neurological examination, and to a behavioral scoring of consciousness with the CRS-R. Our objectives were fourfold: (i) probe the diagnostic reliability of our test at the individual-level on a large sample of well characterized noncommunicating patients with various degrees of consciousness impairments, (ii) estimate its utility in extreme situations such as "locked in syndrome" and related conditions, and (iii) explore in details the distinct ERP correlates of the violations of local and global regularities, both at the group-level and at the individual level, (iv) report the ERP correlates of task learning and stimuli expectancy. Note that the main objective of this study being the validation of the specificity of the ERP "global effect" at the individual level, we deliberately included all ERP datasets originating from various etiologies, recorded either at acute or chronic stages, and we included repeated recordings of the same patients (11 patients with 2-4 recordings) to avoid arbitrary data selection. This study does not aim at reporting specific knowledge about a given disease, of about a specific group of patients, but rather aims at 
testing the value of our ERP test at individual level in regards to the clinical evaluation of consciousness. 


\section{Materials \& Methods}

\subsection{Subjects}

\section{Normal controls}

Experiments were approved by the Ethical Committee of the Salpêtrière hospital. The 10 normal controls (mean age $=20.3 \pm 0.7$; sex-ratio $(M / F)=2.3$ ) gave written informed consent. Data from two control subjects were discarded from the analysis due to excessive movement artefacts.

\section{Patients}

The clinical motivation for recording patients was to better assess their level of consciousness (Bekinschtein, Dehaene et al., 2009), and to probe potential residual unconscious processing of the auditory environment (e.g. : MMN) which predicts consciousness recovery from comatose state (Fischer, Luaute, Adeleine, \& Morlet, 2004 ; Naccache, Puybasset, Gaillard, Serve, \& Willer, 2005). Patients were recorded without sedation since at least 24 hours in order to maximize their arousal and their level of cognitive performance during the auditory task. Among the 100 recordings performed on non-communicating patients, 33 were discarded from the analysis after evaluation of EEG signal quality (see below). This high rate of rejection (33\%) reveals one of the intrinsic limits of this approach. Two other recordings were discarded because they were performed under sedation. The 65 valid datasets included 49 patients (32 males and 17 females, sex-ratio $=1.88)$, aged from 16 to 83 years (mean $=$ $47.5 \pm 17.4$ years). Patients could be recorded from one to 4 times. They were affected by the following usual conditions (see Table for detail): anoxia (35\%), intracranial haemorrhage (28\%), traumatic brain injury (18\%), and other etiologies (18\%). Our dataset included both early and late recordings $($ mean $=203$ days; median $=35$ days; SD $=591$ days; earliest $=6$ days; latest $=2555$ days $)$.

\subsection{Behavioural assessment of consciousness}

Clinical evaluation of consciousness was based on the French version of the CRS-R scale (Schnakers, Majerus et al., 2008), after careful neurological examination by trained neurologists (FF, LN). This scale consists of 23 items that comprise six subscales addressing auditory, visual, motor, oromotor, communication and arousal functions. CRS-R subscales are comprised of hierarchically arranged items. The lowest item on each subscale represents reflexive activity while the highest items represent cognitively-mediated behaviors. This 
scoring enables a distinction to be drawn between conscious (CS), minimally conscious (MCS) and vegetative (VS) states (Schnakers, Vanhaudenhuyse et al., 2009). Clinical examination and behavioural assessment were systematically performed the same day and before EEG recording.

\subsection{Auditory stimulation}

We used the local-global protocol described in our previous publication (Bekinschtein, Dehaene et al., 2009). Series of five complex 50ms-duration sounds were presented via headphones with an intensity of $70 \mathrm{~dB}$ and $150 \mathrm{~ms}$ SOA between sounds. Each sound was composed of three superimposed sinusoidal tones (either a low-pitched sound with 350700 and $1400 \mathrm{~Hz}$ tones, hereafter sound A; or a high-pitched sound with $500 \mathrm{~Hz} 1000 \mathrm{~Hz}$ and $2000 \mathrm{~Hz}$ tones, hereafter sound B). Tones were prepared with $7 \mathrm{~ms}$ rise and $7 \mathrm{~ms}$ fall times. Four different series of sounds were used, the first two using the same five sounds (AAAAA or BBBBB); and the other two with the final sound swapped (either AAAAB or BBBBA). Series of sounds were separated by a variable interval of $1350 \mathrm{~ms}$ to $1650 \mathrm{~ms}$ (50ms steps). The blocks were designed to contain the sound series with a deviant sound in the end, either as an infrequent stimulus (block type a: $80 \%$ AAAAA / 20\% AAAAB; block type b: $80 \%$ BBBBB / 20\% BBBBA); or as a frequent stimulus (block type c: 80\% AAAAB / 20\% AAAAA; block type d: $80 \%$ BBBBA / 20\% BBBBB). All block types presented a local regularity (the fifth sound could be deviant or identical to previous sounds) and a global regularity (one of the series of sounds was less frequent than the other). Each block started with 20-30 series of sounds of the frequent type in order to establish the global regularity with $100 \%$ regular stimuli, before switching to the block with $80 \%$ frequent and $20 \%$ rare stimuli. ERPs elicited by these training trials were used to analyze the learning effect (see below). In each block the number of infrequent trials varied between 22 and 30. All stimuli were presented using Eprime v1.1 (Psychology Software Tools Inc., Pittsburgh, PA). Instructions were delivered auditorily to all patients at the beginning of each block: "You will now listen to repetitive series of 5 sounds. At the beginning of each sequence, you will listen to the very same series which will repeat unchanged. For instance, you will be listening to repetitive series like 'bip bip bip bip bip' or 'bip bip bip bip boop'. Then, after a few tens of seconds you will listen to different series, which will differ from the previous ones. Such new series will remain rare and intermixed with frequent series identical to the initial repetitive series. Each time you will listen to such a different and rare series, we ask you to pay attention to it very carefully, and to count it in your head. Be careful, the sounds will begin in a few seconds". 
All subjects heard eight blocks (3-4 minutes duration) in a fixed order (two runs of AAAAA, BBBBB, AAAAB, BBBBA global standards).

\subsection{High-density scalp ERPs}

ERPs were sampled at $250 \mathrm{~Hz}$ with a 256-electrode geodesic sensor net (EGI, Oregon, USA) referenced to the vertex. Trials were then segmented from $-200 \mathrm{~ms}$ to $+1300 \mathrm{~ms}$ relative to the onset of the first sound. We rejected voltages exceeding $\pm 150 \mu \mathrm{V}$, eye-movements activity exceeding $\pm 80 \mu \mathrm{V}$ and eye-blinks exceeding $\pm 150 \mu \mathrm{V}$. Channels with a rejection rate superior to $20 \%$ across trials were rejected. Bad channels were interpolated. Trials with more than 20 bad channels were rejected. The remaining trials were averaged in synchrony with stimulus onset, digitally transformed to an average reference, band-pass filtered $(0.5-20 \mathrm{~Hz})$ and corrected for baseline over the 800-ms window before fifth sound onset. All these processing stages were performed in the EGI Waveform Tools Package. Analyses of local, global and expectancy effects were done exclusively on the test trials, while the analysis of learning effect included training trials.

\section{Criteria of data quality}

Recording high-density scalp ERPs in ICU, or similar environments, in non-communicating patients is very challenging for technical reasons. First, the electro-magnetic environment is noisy, and patients cannot be recorded in a Faraday cage but necessarily at bedside. Second, many patients present physiological artifacts such as EMG, eye-movements and blinks, or other involuntary movements. Therefore, it is particularly important to systematically evaluate the technical quality of data before statistical analysis. Recordings including at least one block with more than $50 \%$ of rejected trials were discarded from further analyses in order to avoid possible biases across experimental conditions.

\subsection{Statistics}

\section{$\underline{\text { Group analysis }}$}

The significance of group-level ERP effects was estimated through the 3 following approaches:

\section{(i) Triple-threshold t-test based statistics}

Matlab 7.0 (Natick, MA, USA) scripts were used to compute sample-by-sample paired t-tests across subjects. Significance threshold was defined by a triple criterion of: $p \leq .01$ for at least 
10 consecutive samples on a minimum of 20 electrodes. Grand-average ERPs, and voltage topographical maps were performed with Cartool software programmed by Denis Brunet (http://brainmapping.unige.ch/Cartool.htm).

\section{(ii) Regions of interests (ROI) analyses}

MMN (early local effect), P3a and P3b (global effect) effects were also probed by using a ROI approach in which voltages were averaged across a group of contiguous electrodes located in the region where the corresponding ERP effect is known to peak in control subjects. In order to avoid circularity we used an independent dataset, - corresponding to a recently published study using a close auditory oddball paradigm (Pegado et al.)-, and selected contiguous electrodes were MMN, P3a and P3b were best observed (adaptation from 128 electrodes to 256 electrodes EGI scalp nets). Using the EGI (Eugene, Oregon, USA) numbering system, these ROI corresponded to the following groups of contiguous electrodes : MMN (Fz centered : 6781415162122 23) ; P3a (Cz centered : 89458090131132186 257) ; P3b (Pz centered : 100101110118119126128 129). Sample-by-sample t-tests were then performed with a p-value threshold at 0.01 for a minimal duration of 10 successive samples $(40 \mathrm{~ms})$. These ROI were also used to perform ANOVAs between groups of patients, by averaging ROI values in time within a relevant time-window (MMN: 140-180 ms ; P3b: 400-600ms).

\section{(iii) Linear regression approach of scalp topographies}

In order to take advantage of the high-spatial resolution (256 electrodes) of our recordings to detect ERP effects in patients, we complemented the electrode-by-electrode and ROI voltages analyses with a multiple-linear spatial regression approach able to exploit scalp topographies of voltages (Pegado et al.). Each major ERP effect (e.g.: MMN, P3a, P3b) was defined by a 257-values vector corresponding to the averaging of voltages during the relevant timewindow in controls subjects (140-180ms for MMN; 232-368ms for P3a; 400-600ms for P3b). Then for each recording dataset, voltage time series (local effect and global effect) were regressed with a model including the effect of interest and a constant regressor. For each group of patients, distributions of $\beta$ coefficients of interest (e.g.: MMN for local effect, P3b for global effect) were tested against the null hypothesis with a t-test $(\mathrm{p}<0.05)$. 


\section{ERP latencies}

Latencies of each effect were estimated by identifying the earliest significant time sample showing an effect (Luck, 2005), both in the triple-threshold t-test based statistics and in the ROI analyses.

\section{$\underline{\text { Inter-group analyses }}$}

Concerning the inter-group analyses, we are aware of the potential bias related to the fact that our 65 ERP recordings do not correspond to 65 different patients but only to 49 patients, some of whom having been recorded several times in same or different groups. We addressed this issue by performing two analyses. First, we performed ANOVAs on a subset of 49 recordings, by keeping only one recording per patient, corresponding to the best clinical status (see SOM for additional figures on the subset of 49 subjects). Second, we also performed a general linear model analysis able to accommodate with the unbalanced design of the whole dataset, using 2 regressors (subjects group (4 levels), and subjects identity (49 levels)). This analysis took into account the presence of some repeated recordings of the same subjects. Both analyses are complementary and allow avoiding arbitrary data selection.

\section{$\underline{\text { Individual subject analysis }}$}

For individual subject statistics, unpaired t-tests across trials were calculated for each time sample. Significance threshold was defined by a triple criterion: $\mathrm{p} \leq .01$ on a minimum of 5 consecutive samples, on a minimum of 10 electrodes. In order to further assess the power of observed effects, we categorized the significance of the local and global effects for each timesample using a 5-levels p-value scale: <.01, <.005, <.001, <.0005, <.0001. A last correction was then used on each recording in order to increase the specificity of our analyses. Given that local effects begin in controls around $100 \mathrm{~ms}$ after the onset of the fifth tone, all p-values of interest (100ms to $736 \mathrm{~ms}$ after fifth tone onset) superior to the lowest p-values observed in this recording within the baseline time-window $(-800$ to $0 \mathrm{~ms})$ were discarded. Finally when p-values of interest were equal to this minimal p-value, the effect was considered significant only if its duration exceeded the longest duration observed at this p-value level within the baseline time-window. A similar correction was applied for the global effect, with a different time-window of interest (200-736ms). Learning effects time windows were respectively 108- 
$244 \mathrm{~ms}$ after first sound onset (early effect), and 200-736 ms after fifth sound onset (late effect).

\section{$\underline{\text { Permutation statistics }}$}

We further checked the statistical significance of our t-test based triple threshold statistics through Monte-Carlo permutations, both for group-level and individual-level analyses. This procedure is particularly relevant to estimate the statistical significance of effects observed with a signal of unknown distribution (Manly, 1997). For group-level statistics, we calculated the shorter duration of observed effects satisfying our paired t-test thresholds, and then computed random permutations in two surrogate groups with the same dataset, and counted the number of surrogate effects satisfying our criterion (a minimum of 10 consecutive samples with paired t-test $\mathrm{P} \leq 0.01$ on a minimum of 20 electrodes) anywhere in the relevant ERP time window (100-735 ms). The number of permutations was set to 2,000 for most analyses: note that only 255 permutations were computed for the analyses performed on the group 8 controls, in which only $2^{8}=256$ distinct permutations exist. We then computed the observed probability of this criterion (number of surrogate effects per 2,000), and used this proportion as an estimate of the first-order $\alpha$ risk. For individual analyses, the same procedure was used with unpaired t-tests, with 2000 permutations. For each analysis, both at the group-level and at the individual-level, a criterion of $\mathrm{P} \leq 0.05$ on permutation tests was required to consider the effect as significant (see (Naccache et al., 2005) for a recent use of this methodology in an intra-cranial ERP study) . 


\section{Results}

\subsection{Behavioural assessment of consciousness}

Among the 100 recordings, 65 were considered as valid on the basis of our procedure of EEG quality evaluation (see M\&M). These 65 correct recordings corresponded to 49 patients, some of whom were recorded several times (from 1 to 4). This heterogeneous collection of recordings included various levels of clinically assessed conscious states ranging from VS, MCS to overtly conscious, and conscious but paralyzed patients. More precisely, these 65 recordings corresponded to: 24 recordings within VS (37\% of recordings), 28 recordings within MCS (43\% of recordings), and 13 recordings within CS (20\% of recordings). Detailed descriptions of clinical characteristics are reported in the Table. Note that 4 patients presented with severe central or peripheral motor impairments which limited the sensitivity of behavioral evaluations: patient \#37 suffered from a brainstem cystic tumor complicated by a brainstem hemorrhage (MCS, CRS-R = 17), and patient \#44 was in a locked-in syndrome caused by a pontic compression secondary to a right cerebellar hemorrhage associated to a right vertebral dissection (CS, CRS-R = 16). Patients \#43 and \#45 were affected by a severe polyradiculoneuropathy (Guillain-Barré polyradiculoneuritis). However, careful examination was sufficient to reveal behavioural signs of consciousness in these two patients (CRS-R $=16$ for the two patients).

\subsection{Event-related potentials}

We analyzed ERP data by focusing on 4 electrophysiological effects. We first describe the two effects we previously reported (Bekinschtein, Dehaene et al., 2009 ; Faugeras et al., 2011): the (1) "local" and (2) "global" effects. We also report here two new ERP effects: (3) the early cortical processing of sounds, and (4) a learning effect.

For each of these 4 ERP effects, results are reported both at the group level and at the individual level.

\section{'Local Effect': responses to violations of short time-scale regularities}

\section{Group analyses}

We first examined brain responses to violations of local (intra-trial) regularity at the group level, by comparing local-deviant (LD) trials ERPs with local-standard (LS) trials ERPs in 
each of the 4 groups (see Figure 2 bottom panel, and SOM figure for detailed scalp topographies of local and global effects).

In the controls group, we replicated our previous findings (Bekinschtein, Dehaene et al., 2009) by observing 4 main results: 1) the absence of local effect up to $100 \mathrm{~ms}$ after fifth tone onset, 2) a first effect ranging from 124 to $184 \mathrm{~ms}$ with a voltage topography corresponding to a typical MMN, 3) a vertex-centered positivity immediately following this MMN ranging from 232 to $368 \mathrm{~ms}$, and 4) the absence of any later effects. These two effects were found significant both with the triple-threshold t-test based statistics and with the ROI analyses (see $\mathrm{M} \& \mathrm{M})$.

In the conscious patients group, the MMN was present and significant (see Figure 2). A linear regression analysis confirmed the scalp topography of this MMN effect (see M\&M; mean $\beta=$ $0.24 ; p=0.001$; see Figure 2, lower right column). The vertex-centered positivity was visible on scalp topographies and confirmed with the linear regression analysis, but did not reach significance in our 2 other statistical approaches (triple-threshold t-test based statistics, and ROI analyses). No late local effects were observed in this group.

In the MCS patients group, both the MMN, and the following positivity (236-340 ms) were found significant with our 3 statistical approaches (for the linear regression analysis of MMN: mean $\beta=0.17 ; p=0.0015)$, and a late local effect was observed as an anterior centered negativity occurring between $688-736 \mathrm{~ms}$.

In the VS patients group the MMN was not significant according to the triple-threshold statistics, but was confirmed with our 2 additional estimations (for the linear regression analysis: $\beta=0.15$; and $p=0.005$ ). The vertex-centered positivity was significant with our 3 measures and spanned from 260-372 ms. Finally, the late anterior negativity observed in the MCS group was present in VS patients group, from 576-692 ms.

When comparing these four groups, MMN amplitude and significance seemed to be strongly related to the level of consciousness (see Figure 2, right bottom panel): the better the patients were in terms of consciousness status, the larger and the stronger their MMNs were. We first tested the amplitude effect by running a one-way ANOVA, restricted to controls and to the subset of 49 patients with only one recording per patient (see Materials \& Methods), with the group (4) factor on the voltage averaged in space across a ROI centered around Fz and in time across a [140-180ms] temporal window. This analysis revealed a main effect of the group factor $(\mathrm{F}(3,54)=4.8 ; \mathrm{p}=0.005)$, and a linear contrast confirmed that MMN amplitudes increased with higher levels of consciousness $(\mathrm{F}(1,54)=13.9 ; \mathrm{p}=0.0005$ for the linear 
contrast testing the following order in MMN amplitude: Controls > Conscious patients > MCS patients > VS patients). We also tested the same amplitude effect on the whole dataset of recordings by running a general linear model analysis with two regressors, including group factor and subject identity factor (see Materials \& Methods). This analysis replicated the modulation of MMN amplitude across groups $(\mathrm{F}(3,68)=3.2 ; \mathrm{p}=0.03)$.

We also estimated and compared MMN latencies across groups. When first analyzing the triple-threshold data, MMN latency was measured at $124 \mathrm{~ms}$ after fifth tone onset in controls, and it was delayed to $140 \mathrm{~ms}$ both in conscious and in MCS patients. Given that MMN was not significant at the group-level for the VS patients in our triple-threshold t-test based statistics, we also estimated latencies through the ROI analysis. In this more sensitive analysis, MMN latencies were estimated at $104 \mathrm{~ms}, 144 \mathrm{~ms}, 152 \mathrm{~ms}$ and $152 \mathrm{~ms}$ respectively for controls, CS patients, MCS patients and VS patients. Note however that a one-way ANOVA performed on individual MMN latencies did not reveal any significant differences across the 4 groups ( $>0.5$ both for the main effect, and for a linear contrast respecting clinical progression).

\section{Individual analyses}

MMN was observed in 7/8 controls $(87.5 \%)$, in 8/13 recordings of CS patients $(61.6 \%)$, in 9/28 recordings of MCS patients (32.1\%), and in 6/24 recordings of VS patients (25\%) (see Figure 3). MMN presence was therefore affected by consciousness status (logistic regression test with the 4 groups declared as a predictor of MMN presence: $p=0.0005$ ). This effect was still present but more modest when restricting the analysis to the 3 patients groups $(\mathrm{p}=0.04)$. A trend of a relation was observed between the CRS score and the presence of a MMN (mean CRS for $\mathrm{MMN}+=12.3$ and mean CRS for MMN- = 9.5; Student t-test unilateral $\mathrm{p}$ value $=$ 0.04). While MMN presence was not different between MCS and VS patients groups ( $\mathrm{p}=0.6$ in a $\chi 2$ test), MMN was statistically more significant in MCS patients than in VS patients (see Figure 3). Indeed, an ANOVA with two orthogonal factors: Group (4) X Local_regularity (2) performed on the voltage averaged in space across a ROI centered around Fz and in time across a [140-180ms] temporal window revealed that MMN amplitude increased with higher levels of consciousness $(\mathrm{F}(1,138)=4.9 ; \mathrm{p}=0.03$ for the linear contrast testing the following order in MMN amplitude: Controls > Conscious patients > MCS patients > VS patients).

\section{The 'Global Effect': responses to violations of long time-scale regularities}

\section{Group analyses}


In the controls, a large global effect was visible on the global field power (GFP) plots, on averaged ERP curves and on scalp topographies (see SOM Figure), and was confirmed with our statistical criterion (see Figure 2, top panels). This global effect spanned from 284 to $660 \mathrm{~ms}$ after the onset of fifth tone, in close agreement with our previous findings. This effect presented a clear P3 topography, beginning with a vertex-centered positivity (P3a) and followed by a more posterior Pz-centered positivity (P3b). Note that before this large and late P3 complex, an earlier and shorter global effect was observed within the MMN window of the local effect, with a similar topography. On the basis of our previous findings which related the large and sustained P3b response to consciousness of the global rule, we focused on this ERP component, and performed a ROI analysis. Voltages were averaged for each control subject across a set of electrodes surrounding $\mathrm{Pz}$ (see upper left panel on Figure 2), and a sample-bysample t-test was performed (see M\&M). P3b latency in controls was estimated at $448 \mathrm{~ms}$ after the fifth sound with the ROI analysis. Note that the triple-threshold statistics revealed an earlier effect (284 ms) corresponding to a P3a effect as mentioned above. A dedicated Czcentered similar ROI analysis estimated P3a latency at $320 \mathrm{~ms}$ in controls.

In conscious patients, neither the triple-threshold statistics nor the ROI analysis could identify a P3b global effect. Nor could the earlier global effect be observed in this group. We then ran the regression analysis to capture a significant P3b effect in this group on the basis of scalp topography information (see above). While visual inspection revealed a scalp topography reminiscent of the P3b on grand-average data (see Figure 2, lower right topographies), the statistical distribution of individual $\beta$ values of the $\mathrm{P} 3 \mathrm{~b}$ regressor did not differ from 0 ( $p>0.4$ ). For the two other groups (MCS and VS patients) none of these 3 analyses could isolate a significant global effect at the group level.

We could then test for the inter-group differences by running an ANOVA, - restricted to controls and to the subset of 49 patients -, with a group (4) factor, on the global effect voltages averaged across the Pz ROI during the P3b time-window. This analysis confirmed the existence of a strong modulation of $\mathrm{P} 3 \mathrm{~b}$ with conscious status $\left(\mathrm{F}(3,54)=14.7, \mathrm{p}<10^{-5}\right)$. This modulation followed the order of consciousness: Conscious controls > Conscious patients > MCS patients $>$ VS patients (linear contrast: $F(1,54)=40.9, p<10^{-5}$ ). A general linear model analysis performed on the whole dataset of recordings replicated this effect $(F(3,68)=6.5$; $\mathrm{p}=0.0006)$.

\section{Individual analyses}


We observed a significant global effect in each of the 8 controls (100\%) within the 300-700 ms temporal window after $5^{\text {th }}$ sound onset (see Figure 4), replicating our previous findings (see our control group \#1 in (Bekinschtein, Dehaene et al., 2009)). In the patients groups, the proportions of individuals showing a significant global effect fell respectively to 7/13 (53.8\%) recordings of CS patients, 4/28 (14.3\%) recordings of MCS patients, and 2/24 (8\%) recordings of VS patients. These differences were highly significant across groups, - as in above the group level analysis - , ( $p<10^{-7}$ in a logistic regression test). An additional analysis confirmed the strong relation prevailing between the CRS score and the presence of a global effect (mean CRS for GE $+=14.7$, and mean CRS for GE- $=9.5 ; \mathrm{p}=0.002$ in a two-sample ttest).

Of particular interest are the VS patients results, we previously reported in a dedicated publication (Faugeras et al., 2011). First, the vast majority of VS patients did not show global effect (2/22). Most notably, only two VS patients showed a global effect, and for both of them the immediate clinical evolution was marked by the recovery of behavioral signs of consciousness within 3 and 4 days after EEG recordings, respectively (see Patient \#1 and Patient \#4's second recording in Table). These two cases are reminiscent of recent reports of the few patients clinically assessed as VS, - on the basis of a detailed clinical examination and CRS scoring -, who showed evidence of consciousness in active fMRI paradigms (Monti et al., 2010; Owen et al., 2006 ). Similarly, our two observations may correspond to such a situation in which neurophysiological probing of consciousness may go beyond clinical evaluation. In favor of such an interpretation note that by contrast, in the 20 remaining VS patients without an ERP global effect, early recovery of consciousness was observed in only 2 cases within the first week following ERP recording $(\chi 2=9.90, \mathrm{p}=0.002$; Exact Fischer test: $\mathrm{p}=0.026$; see (Faugeras et al., 2011) for a detailed report of the VS patients group).

Thus, the global effect proved to be an almost $100 \%$ specific test of consciousness. Even if the 2 VS patients showing a global effect and who recovered behavioral signs of consciousness are classified as "false negative" subjects we obtain the following values: specificity (correct rejections/(false positives+correct rejections $)=91.7 \%$; positive predictive value $($ hits $/$ (hits+false positives $)=84.6 \%$; sensitivity $($ hits $/$ (hits+false negatives $)=26.8 \%$; and negative predictive value (correct rejections/(correct rejections + false negatives) $=42.3 \%$. Alternatively, if we classify these 2 VS patients as conscious subjects, then we obtain a specificity and a positive predictive value of $100 \%$. 


\section{Early cortical processing of sounds}

We then explored ERPs prior to the processing of local and global regularities. Given the strong differences in both local and global effects in relation to conscious status of patients, we wondered if even earlier differences could be observed.

\section{Group analyses}

We inspected responses to auditory sounds across a region of interest defined by the 23 electrodes surrounding the vertex electrode $(\mathrm{Cz})$, where early cortical responses to sounds are best observed (see Figure 5). For each recording of the subset of 49 patients, we averaged ERPs around $\mathrm{Cz}$ across all global standard trials, applied a 200ms baseline correction, and submitted these values to a sample-by-sample one-way ANOVA with the group (4) factor. A cortical response to each of the first four sounds was visible in the 4 groups (see Figure 5, top panel). Interestingly, early cortical processing of the sounds was not affected by clinical status up to $\sim 250 \mathrm{~ms}$ after the onset of the first of the five sounds defining each trial. This finding is highly consistent with the large group of studies reporting spared early non-conscious processing in comatose or VS patients, and consequently confirms the need to disentangle between neural markers of conscious versus non-conscious processing. From $\sim 250 \mathrm{~ms}$ up to $\sim 1100 \mathrm{~ms}$ after the onset of the first sound, significant differences were observed across groups ( $\mathrm{p}$ values $<0.05$; a very same pattern of significance was observed when analyzing the whole recordings dataset using a general linear model analysis taking into account subject identity). These inter-group differences corresponded mostly to a difference in the strength of a negative drift ending during the processing of the fifth sound which is the event defining the nature of the trial (local and global regularities). While this negative drift was visually obvious for controls, and to a lesser degree for conscious patients, it seemed less present for the two other groups of patients. For each group, and also for each individual recording, we computed the linear regression of these averaged ERPs within the [0-600 ms] time-window running from the onset of the first sound up to the onset of the fifth sound. This analysis confirmed the existence of a significant negative slope for controls $\left(\mathrm{R}^{2}=0.64\right.$; all individual slopes were negative; t-test $\mathrm{p}$ value $<10^{-4}$ when comparing slopes distribution to a zerocentered distribution) and for conscious patients $\left(\mathrm{R}^{2}=0.45\right.$, all individual slopes were negative; t-test $\mathrm{p}$ value $\left.<10^{-4}\right)$, while this effect was absent for the two other groups $\left(\mathrm{R}^{2}=0.10\right.$ $\mathrm{p}=0.1$ for MCS patients; and $\mathrm{R}^{2}=0.26 \mathrm{p}=0.06$ for VS patients). An ANOVA performed on controls data and on the subset of 49 patients confirmed the significance of slope differences 
across the 4 groups $(F(3,54)=5.0, p=0.004)$. The general linear model analysis ran on the whole dataset showed a similar, but weaker, effect $(\mathrm{F}(3,68)=2.7 ; \mathrm{p}=0.05)$.

This slow ERP effect is suggestive of the classical 'Contingent Negative Variation' (see (Walter, Cooper, Aldridge, McCallum, \& Winter, 1964) and discussion section) on the basis of 3 arguments: 1) it is an early effect beginning with the early perceptual processing of the first sound, - which is probably reflecting an expectancy of the delivery of the fifth sound, the sound that conveys the critical information about local and global regularities of the trial -, and ending when this last sound is processed ; 2) it presents an anterior negativity scalp topography reminiscent of the $\mathrm{CNV}$; and 3) it is observed exclusively in the groups of clearly conscious subjects (controls and CS groups), more prone to deploy expectative cognitive processes than the two other groups. Therefore, we will now refer to this early negative drift effect as a CNV.

\section{Individual analyses}

At the individual level, a linear regression was calculated for each individual trial on the vertex centered ROI during the [0-600 ms] time-window of interest. Then the distribution of these individual trials slopes was compared to zero with a one-sample Student t-test. A significant CNV was observed in each of the 8 controls (100\%), in 8/13 (61\%) recordings of of conscious patients, in 12/28 (43\%) recordings of of MCS patients, and in 9/24 (37\%) recordings of VS patients (see Figure 5, middle panel). In patients, CNV was not more present in MCS or conscious state recordings than in VS recordings ( $\mathrm{p}=0.4$ in a $\chi^{2}$ test). Most of recordings showing a global effect had a CNV (61\%). However, the presence of a CNV was not strongly associated with global effect ( $p=0.2$ in a $\chi^{2}$ test). Recordings with a CNV were slightly more prone to present a MMN ( $\mathrm{p}=0.06$ in a $\chi^{2}$ test). In order to determine the impact of CNV presence on local and global effects at the group level, we then categorized each recording either as $\mathrm{CNV}+$ or as $\mathrm{CNV}$-, and computed local and global effects in these two groups. If the $\mathrm{CNV}$ does index cognitive expectations of stimuli regularities, one should predict that $\mathrm{CNV}$ presence should increase the likelihood of observing other markers of the processing of auditory regularities. In particular, under such a hypothesis both global effect and late local effect should be more important in $\mathrm{CNV}+$ recordings than in $\mathrm{CNV}$ - recordings. Local effects did not differ between CNV+ and CNV- groups (see Figure 5, bottom panel). In particular, MMN, P3a and late local effect were present and comparable (all $\mathrm{p}$ values $>0.5$ on t-tests performed on $\mathrm{Fz}$ ROI during MMN time window, on $\mathrm{Cz}$ ROI during the positivity 
immediately following the MMN, and during the late local effect). In sharp contrast, global effect was observed only in the CNV+ group, while it was absent in the CNV-group.

\section{Learning effect}

Finally, we looked for learning effect by taking advantage of recording the EEG signal both during the training and testing periods. Each block began with the delivery of verbal instructions followed by 20-30 series of frequent type in order to establish the global regularity with $100 \%$ regular stimuli. Then, the testing period followed without interruption, with $80 \%$ series of the frequent type ("global standards"), and $20 \%$ of the rare type ("global deviants"). While all previous analyses focused on the testing period, we compared here the processing of the very same physical stimuli ("global standards"), according to the period during which they were processed (learning versus testing periods).

\section{Group analyses}

In controls, this comparison revealed two learning effects visible on the anterior, Fz-centered, region: first, an anterior positivity (testing trials minus training trials) occurring around 108$244 \mathrm{~ms}$ after first sound onset, followed by a delay effect presenting like an anterior negativity occurring around 312-620 ms after fifth sound onset (see Figure 6). This second effect was statistically weaker.

In the conscious patients group only the second effect was observed, while none of these 2 effects was present in MCS and VS patients groups.

The first effect was also observed when contrasting global standard trials from the learning period with global deviant trials from the testing phase. Indeed, the time-window of this effect is located before the onset of the fifth sound. Obviously, such an analysis is not relevant for the late effect which overlapped with the global effect.

\section{Individual analyses}

In controls, $4 / 8$ showed the first effect, while $2 / 8$ showed the late effect. Taken together $5 / 8$ conscious controls showed a learning effect. In conscious patients, 2/13 recordings showed the early effect, and 5/13 showed a significant late effect. Among MCS patients, 5/28 recordings showed the early effect, and 2/28 the late effect. Finally within VS patients, only one recording showed the early effect, and another patient had a late effect. Interestingly, the clinically 'VS' patient with the early learning effect was one of the two who presented a 
global effect and who were probably misclassified and conscious during the recording (see above and discussion, and (Faugeras et al., 2011)).

\section{Discussion}

In this work we prospectively recorded high-density scalp ERP in patients suffering from various disorders of consciousness, while they were instructed to perform an active version of the 'local global test'. This test was recently designed to diagnose consciousness without relying on behavioral responses. Among these 100 recordings, 65 satisfied criteria of data quality, due to the large amount of motor and environment EEG artifacts. Both the clinical condition of patients who were recorded in the absence of any sedative drugs, - enabling therefore motion artifacts -, and the noisy electrical environment inherent to intensive care units most probably contribute to explain this high-rate of data rejection. The acceptable recordings correspond to 49 patients, some of whom were recorded several times.

\subsection{Strength of the global effect for probing consciousness: a positive predictive value close to $100 \%$}

The presence of an ERP global effect proved to be an extremely specific measure of consciousness: among the 13 recordings showing a global effect, 11 corresponded to conscious patients (MCS or conscious states) and only 2 were observed in clinically diagnosed VS patients. Crucially, in these 2 patients recorded during the acute phase of their consciousness disorder (see Table, patient \#1 and second recording of patient \#4 on line 14), univocal behavioral signs of consciousness were present respectively 3 and 4 days after recording (see (Faugeras et al., 2011)). In other terms, these two observations most probably illustrate situations in which neurophysiological data can go beyond the sensitivity of behavioral measures to probe consciousness, as it was recently reported with an active mental imagery task in fMRI ((Monti et al., 2010; Owen et al., 2006)). In addition to these two extreme cases, we could also demonstrate the usefulness of this test in 2 conscious patients affected with a severe acute polyradiculoneuropathy (Guillain-Barré syndrome) who showed a clear global effect confirming the clinical diagnosis (see Table, patient \#45 and patient \#43) of consciousness. In these two patients, behavioral signs of consciousness were subtle and called for careful inspection. For instance, in one of these patients, the only voluntary movements were limited to discrete lateral flexions of the neck initially discovered by an expert ICU neurologist (FB). As a provisional conclusion the 'local global' test demonstrated 
its clinical robustness and its positive predictive value to probe consciousness in noncommunicating patients. Of major interest, this test seems powerful enough to provide reliable measures at the individual level.

Our finding that the global effect is observed exclusively in conscious patients is equally important for theories of consciousness. The 'local global' test is based on the global workspace theory of conscious processing which postulates that consciousness is required to actively maintain perceptual information over time in working memory, and to engage strategical processes necessary to detect and count the global deviance occurrences (Bekinschtein, Dehaene et al., 2009; Dehaene \& Naccache, 2001 ). We previously showed that in conscious controls the global effect is abolished when attention is captured by a concurrent visual task. In this experimental condition (see Experiment 3 of (Bekinschtein, Dehaene et al., 2009)), subjects could not consciously report the presence of a global regularity after the recording. However, one could have imagined that the limiting factor in this case was not consciousness per se, but rather the availability of cognitive resources engaged by top-down conscious processing in another task. In other terms, an important complementary condition is to demonstrate that the global effect does not occur in non conscious patients in whom residual cognitive abilities are not engaged in an active distracting task. The present study strengthens the causal relation prevailing between consciousness and the cognitive processes at work in the 'local global' paradigm. Our work therefore confirms that active maintenance of perceptual information during an arbitrary task requires consciousness. Additionally our results also reinforce the set of recent studies proposing that late P3b-like ERP scalp topographies may constitute a specific neural signature of conscious access (Gaillard et al., 2009; Sergent, Baillet, \& Dehaene, 2005 ).

\subsection{Limits of the global effect: a low negative predictive value}

In spite of its high specificity and positive predictive value, the global effect presents a much less satisfactory sensitivity $(\sim 27 \%)$ and negative predictive value $(\sim 42 \%)$, indicating that many patients easily classified as conscious by clinical criteria do not show a significant global effect in ERPs. Several factors may concur to explain this lack of sensitivity. First, auditory processing prior to cortical stages has to be preserved to allow the presence of a global effect (obviously, a deaf conscious patient would not show any global effect). Careful clinical examination (sound localization responses, auditory startle response) and inspection of preserved P1/N1 to auditory stimuli are strong arguments to discard such an explanation. Note that in the presence of any doubt of deafness, brainstem auditory evoked potentials 
(BAEP) are to be recorded. Second, the presence of a global effect not only requires the patient to be awake and conscious during the recording, but also that he/she would understand the instructions, be able to keep perceptual representations in working memory, memorize task instructions and continuously perform the task. In other terms, a conscious patient affected with cognitive impairments in any of the listed processes may perfectly well miss the task. Our group analyses suggest that in such cases, a conscious patient unable to perform the global task may well process consciously the local regularity, as shown by the presence of late local effects in the MCS and VS groups, while both conscious controls and conscious patients presenting a global effect did not show any late local effect. On the other side, the presence of an ERP global effect may well be considered as the sign of a rich conscious cognition, and could prove to be a useful index of cognitive outcome. Future outcome studies should elucidate this point. The limits exposed here are inherent to many active cognitive paradigms (Naccache, 2006; Owen et al., 2006 ). In the same vein, fluctuations of attention or arousal may impair task performance. Some of these limitations could be overcome by sorting individual trials on the basis of additional EEG measures more related to arousal or attention (e.g. : proportion of slow waves, measures of coherence and/or EEG complexity indexes such as EEG entropy or EEG dimensional activation (Velly et al., 2007)). The current emergence of brain-computer interfaces allowing such analyses to be performed in real-time on a singletrial basis may well significantly increase the sensitivity of our test (Delorme \& Makeig, 2004; Thulasidas, Guan, \& Wu, 2006). Note that in spite of its low sensitivity, this test is of medical interest because clinical consensus on patients' consciousness status is often erroneous, as recently reported by Schnakers and colleagues (2009): "Of the 44 patients diagnosed with VS based on the clinical consensus of the medical team, 18 (41\%) were found to be in MCS following standardized assessment with the CRS-R." In other terms, when focusing on the VS and MCS patients who are difficult to classify reliably on standard clinical measures, - without fine behavioral measures such as the CRS-R -, it is not absolutely useless to find 6 positive recordings out of 52. ERPs added some confidence for the MCS patients, and corrected the functional diagnosis in two clinically VS patients.

\subsection{ERP responses to violations of local regularities}

Violations of local regularities elicited three successive ERP effects, the occurrence of which differed across groups. First, a classical MMN response was observed within 140-180 ms after fifth tone onset. Interestingly, MMN was affected by clinical status: it was larger, better delineated (scalp topography), and more significant in the groups of conscious controls and 
conscious patients than in MCS and VS patients groups. This result is in agreement with a recent study reporting a progressive increase of MMN quality during the VS to MCS transition in a few individual patients (Wijnen, van Boxtel, Eilander, \& de Gelder, 2007). Given that early MMN (90-160 ms) is a pre-attentional automatic ERP component impermeable to conscious top-down processes (Naatanen, Tervaniemi, Sussman, Paavilainen, \& Winkler, 2001 ; Pegado et al.; Tiitinen, May, Reinikainen, \& Naatanen, 1994 ), and given that it has been reliably observed in comatose and VS patients (Bekinschtein, Dehaene et al., 2009; Fischer, Luaute, Adeleine, \& Morlet, 2004; Fischer et al., 1999 ; Kane, Curry, Butler, \& Cummins, 1993 ; Naccache, Puybasset, Gaillard, Serve, \& Willer, 2005 ), the finding that even early MMN is impaired in MCS and VS patients indicate that these patients are not characterized by 'pure' impairments of consciousness, but that they most probably combine arousal and perceptual deficits to disorders of consciousness. This highly plausible interpretation is supported by recent works questioning the definition of VS as a pure dissociation between a preserved arousal function, and an impaired consciousness. For instance, Bekinschtein and colleagues (2009) showed that while VS patient are able to keep their eyes open spontaneously, they do not show normal circadian rhythms of body temperature or other physiological markers. Therefore, the combination of neurophysiological markers exploring both conscious processing (e.g.: P3b global effect response) and unconscious perceptual abilities (e.g.: early local effects) may help to better estimate the range of preserved cognitive faculties. In this perspective, the design and use of multifaceted ERP batteries exploring distinct cognitive processes in the same patient would be promising. For instance, the combination of paradigms probing verbal semantic processing ( $\mathrm{N} 400$ word priming experiments (Rama et al.)), verbal syntactic decoding (P600 sentence paradigms (Kotchoubey, 2005)), and emotional processing of meaningful stimuli (e.g.: processing of known voices or own name (Holeckova, Fischer, Giard, Delpuech, \& Morlet, 2006)), in addition to MMN and markers of conscious access may prove useful both for diagnostic and cognitive prognosis issues. The present study reinforces the relevance of this approach.

Second, immediately following the MMN response we observed an anterior positivity response which may well correspond to a P3a response indexing a central stage of processing of local deviance. Indeed, the finding that this component was less significant both in the groups of control subjects and in conscious patients than in the two other patients groups may be interpreted in terms of limited central capacity: when subjects do perform the global deviance detection task they have to process this attribute without responding to the local deviance, which is in opposition to global deviance in half of the trials. Therefore, given the 
limited serial property of conscious content, they would be able to consciously process only a single dimension (global deviance). As a consequence, the ERP component following the MMN would be predicted to be smaller in such conscious subjects performing the task, than both in non-conscious patients in whom only automatic responses to local deviance would be observed, and in conscious but cognitively impaired patients whom central resources would be captured by the processing of local deviance. This hypothesis is strengthened by two elements: the anterior positivity following the MMN in the local effect contrast (local deviant versus local standard trials) overlap in time with the beginning of the P3 complex observed in the global effect contrast (global deviant versus global standard trials). Moreover, we recently provided evidence in support of a two-stage model of accumulation of evidence during auditory oddball paradigms (Pegado et al.). According to this model and experimental data, late portion of the MMN (160-220 ms) and subsequent components (including the following positivity) are interpreted as central stages of processing. Assuming that this interpretation is valid, and that the absence of a P3a response to local deviance in conscious subjects reflect their conscious engagement in the processing of an orthogonal dimension of the stimuli (global deviance), one may well understand the presence of this ERP component in the MCS group (conscious processing of local deviance due to cognitive impairments), but may wonder why this ERP component would be observed in the VS patients group? While speculative, we may propose that this ERP component could reflect a form of unconscious exogenous attention which may be preserved in some non conscious patients, and which would not be an index of reportable conscious contents. Indeed, in conscious controls an increasing set of evidence point to the existence of such unconscious deployment of attentional resources, for instance in response to salient stimuli (Koch \& Tsuchiya, 2007; Mulckhuyse \& Theeuwes, $2010)$.

Lastly, a clear difference across the four groups was the presence of a late anterior negativity in the MCS and VS patients groups, while no late effect was observed in the two conscious subjects groups (controls and conscious patients). Interestingly, we previously observed such a late effect both in one MCS patient, and in several conscious controls under an attentional manipulation preventing them from being conscious of the global regularity (see groups 2 and 3 of (Bekinschtein, Dehaene et al., 2009), and patient MCS\#4 in the same study). We proposed that such a late local effect could reflect that these individuals: "processed consciously the local deviant trials, yet without being able to detect the existence of a global regularity". In other terms, we hypothesized that given the limited capacities of conscious access (Dehaene \& Naccache, 2001), a subject who is representing consciously 
representations of stimuli in terms of global deviance (e.g.: conscious control subject) would not represent consciously local deviance of stimuli, and therefore would not show late local effects. In contrast, a conscious subject unable to represent global deviance could show such late local effects. This line of interpretation of this effect would make use of the very same logic as for the P3a response (see above). This component may therefore reflect deeper cognitive integration of the local deviance than MMN. Further studies may use this late local effect to better discriminate across VS patients those with deeper perceptual integration of the environment.

\subsection{An early ERP marker of cognitive expectations}

While this work was driven by the exploration of ERP responses to both global and local deviances of the stimuli, we discovered an unexpected ERP response. We observed that prior to the delivery of the fifth sound, - which was conveying simultaneously informations of local and global deviance of the current trial -, conscious controls showed a clear slow negative drift beginning with the onset of the first tone of the series, and closing with the early ( 200 ms) processing of the fifth tone. As we noted above (see Results section) this ERP effect is extremely suggestive of the classical 'Contingent Negative Variation' (Walter, Cooper, Aldridge, McCallum, \& Winter, 1964) which has been initially described in a paradigm where the subjects are instructed that a warning stimulus followed by a target stimulus will be delivered on each trial, with a random or fixed stimulus onset asynchrony (SOA). Processing of the warning stimulus elicits a CNV which terminates when the target stimulus is delivered and processed. $\mathrm{CNV}$ has been reported in various motor and cognitive tasks, and has been reported to correspond to the activation of a large network implicating in particular frontal cortices (Gomez, Flores, \& Ledesma, 2007 ; Niedermeyer, 2003 ; Rosahl \& Knight, 1995). $\mathrm{CNV}$ is affected by manipulations of attention and motivation (Rockstroh, Elbert, Birbaumer, \& Lutzenberger, 1982). In the present study, the negative drift showed an anterior negativity scalp topography reminiscent of the $\mathrm{CNV}$, and the regular structure of our stimuli encouraged expectative processes of the fifth sound once the first sound was perceived. As in classical 'S1-S2' CNV paradigms, a first stimulus announced the occurrence of the second and crucial stimulus on which a task had be performed (Walter, Cooper, Aldridge, McCallum, \& Winter, 1964). Moreover, we could identify this CNV component only in groups of conscious subjects (controls and patients), further corroborating the cognitive nature of this ERP component. At the individual level, we could isolate a $\mathrm{CNV}$ in all conscious controls, and in the majority 
(61\%) of conscious patients' recordings. Capitalizing on these premises, we then sorted all patients' recordings according to the presence of this $\mathrm{CNV}(29 \mathrm{CNV}+$ and $36 \mathrm{CNV}$ recordings). We then recomputed the local and global effects in each of these two groups. While both groups showed early and late local effects, we observed a clear global effect in the $\mathrm{CNV}+$ group only. Note that this is not a trivial result given that no global effect could be observed at the group level in any of the 3 groups of patients. As discussed about the late local effect, this CNV component may help to distinguish among MCS and VS patients those who display a richer mental life. At a theoretical level, relations prevailing between $\mathrm{CNV}$ and consciousness are not very well documented. As far as we could review the literature, a single observation on two comatose patients reported the presence of a component resembling CNV (Dolce \& Sannita, 1973). By reporting here experimental data showing that multiple VS patients displayed a clear $\mathrm{CNV}$ in the absence of any clinical and electrophysiological markers of consciousness, we provide strong evidence in favor of the existence of nonconscious expectative processes. As for the exogenous deployment of attention by salient stimuli, it seems that expectative processes, driven by auditory regularities delivered within a short time-window compatible with unconscious echoic memory system, exist. One limitation of our study relies in the fact that both local and global deviance informations were conveyed by the same event. Therefore, it is not easy to distinguish between local and global regularities expectations, and in consequence between conscious and unconscious forms of expectation. Future works may use this $\mathrm{CNV}$ approach to distinguish between conscious and nonconscious forms of expectations, and to test for their independence.

\subsection{Learning effects}

This paradigm also offered us the opportunity to look for learning effects by contrasting ERPs elicited by the very same stimuli, according to the period during which they were delivered. Interestingly, these effects could reflect attentional and/or strategical learning effects. Indeed, they are not confounded with time given that the learning periods were distributed over the 8 experimental blocks. Note also that the first learning effect occurring well before the onset of the fifth sound cannot be explained simply by a difference in probabilities of the first sounds which were identical across both conditions. Moreover, the observation that only conscious and MCS subjects showed this early learning effect is coherent with an attentional/strategical interpretation. Indeed, the single VS patient showing this early effect was probably conscious during recording: she presented both a learning and a global effects, and recovered univocal signs of consciousness 3 days after the recording session (see (Faugeras et al., 2011)). It is 
noteworthy that when considering the presence of either the global effect or the early learning effects, one can discriminate conscious patients (conscious and MCS patients) from VS patients with a similar and almost perfect specificity (see above), and with a stronger sensitivity (39\% versus $27 \%$ ) than when using the global effect alone. It is possible that spectral analyses enrich the detection of these learning effects which may correspond to sustained cognitive states rather than to events locked in time with stimuli or responses. Future analyses may confirm this hypothesis.

\subsection{Conclusion}

The ERP 'global effect' can be used as a highly specific marker of consciousness in noncommunicating patients with a specificity close to $100 \%$. In the presence of a global effect in an individual clinically diagnosed as non-conscious (e.g.: VS), one has to question the clinical diagnosis and to carefully observe the patient. Future works taking advantage of real-time analysis of EEG signal, and combining several electrophysiological measures of brain activity may increase the sensitivity of this index of consciousness and may go beyond diagnosticoriented measures of brain activity, to enable communication in conscious but impaired patients through real-time brain computer interfaces. 


\section{References}

Bekinschtein, T. A., Coleman, M. R., Niklison, J., 3rd, Pickard, J. D., \& Manes, F. F. (2008). Can electromyography objectively detect voluntary movement in disorders of consciousness? J Neurol Neurosurg Psychiatry, 79(7), 826-828.

Bekinschtein, T. A., Dehaene, S., Rohaut, B., Tadel, F., Cohen, L., \& Naccache, L. (2009). Neural signature of the conscious processing of auditory regularities. Proc Natl Acad Sci U S A, 106(5), 1672-1677.

Bekinschtein, T. A., Golombek, D. A., Simonetta, S. H., Coleman, M. R., \& Manes, F. F. (2009). Circadian rhythms in the vegetative state. Brain Inj, 23(11), 915-919.

Bekinschtein, T. A., Shalom, D. E., Forcato, C., Herrera, M., Coleman, M. R., Manes, F. F., et al. (2009). Classical conditioning in the vegetative and minimally conscious state. Nat Neurosci, 12(10), 1343-1349.

Bernat, J. L. (2006). Chronic disorders of consciousness. Lancet, 367(9517), 1181-1192.

Blumenfeld, H., \& Taylor, J. (2003). Why do seizures cause loss of consciousness? Neuroscientist, 9(5), 301-310.

Clark, R. E., \& Squire, L. R. (1998). Classical conditioning and brain systems: the role of awareness. Science, 280(5360), 77-81.

Coleman, M. R., Davis, M. H., Rodd, J. M., Robson, T., Ali, A., Owen, A. M., et al. (2009). Towards the routine use of brain imaging to aid the clinical diagnosis of disorders of consciousness. Brain, 132(Pt 9), 2541-2552.

Dehaene, S., Changeux, J. P., Naccache, L., Sackur, J., \& Sergent, C. (2006). Conscious, preconscious, and subliminal processing: a testable taxonomy. Trends Cogn Sci, 10(5), 204-211.

Dehaene, S., \& Naccache, L. (2001). Towards a cognitive neuroscience of consciousness: basic evidence and a workspace framework. Cognition, 79(1-2), 1-37.

Delorme, A., \& Makeig, S. (2004). EEGLAB: an open source toolbox for analysis of singletrial EEG dynamics including independent component analysis. J Neurosci Methods., 15(134), 9-21.

Dolce, G., \& Sannita, W. (1973). A CNV-like negative shift in deep coma. Electroencephalogr Clin Neurophysiol, 34(6), 647-650.

Driver, J., \& Mattingley, J. B. (1998). Parietal neglect and visual awareness. Nat Neurosci, l(1), 17-22.

Faugeras, F., Rohaut, B., Weiss, N., Bekinschtein, T. A., Galanaud, D., Puybasset, L., et al. (2011). Probing consciousness with event-related potentials in the vegetative state. Neurology, 77(3), 264-268.

Fischer, C., Luaute, J., Adeleine, P., \& Morlet, D. (2004). Predictive value of sensory and cognitive evoked potentials for awakening from coma. Neurology, 63(4), 669-673.

Fischer, C., Morlet, D., Bouchet, P., Luaute, J., Jourdan, C., \& Salord, F. (1999). Mismatch negativity and late auditory evoked potentials in comatose patients. Clin Neurophysiol, 110(9), 1601-1610.

Gaillard, R., Dehaene, S., Adam, C., Clemenceau, S., Hasboun, D., Baulac, M., et al. (2009). Converging intracranial markers of conscious access. PLoS Biol, 7(3), e61.

Giacino, J. T., Ashwal, S., Childs, N., Cranford, R., Jennett, B., Katz, D. I., et al. (2002). The minimally conscious state: definition and diagnostic criteria. Neurology, 58(3), 349353.

Gomez, C. M., Flores, A., \& Ledesma, A. (2007). Fronto-parietal networks activation during the contingent negative variation period. Brain Res Bull, 73(1-3), 40-47.

Holeckova, I., Fischer, C., Giard, M. H., Delpuech, C., \& Morlet, D. (2006). Brain responses to a subject's own name uttered by a familiar voice. Brain Res, 1082(1), 142-152. 
Jennett, B., \& Plum, F. (1972). Persistent vegetative state after brain damage. A syndrome in search of a name. Lancet, 1(7753), 734-737.

Kalmar, K., \& Giacino, J. T. (2005). The JFK Coma Recovery Scale--Revised. Neuropsychol Rehabil, 15(3-4), 454-460.

Kane, N. M., Curry, S. H., Butler, S. R., \& Cummins, B. H. (1993). Electrophysiological indicator of awakening from coma. Lancet, 341(8846), 688.

Koch, C., \& Tsuchiya, N. (2007). Attention and consciousness: two distinct brain processes. Trends Cogn Sci, 11(1), 16-22.

Kotchoubey, B. (2005). Event-related potential measures of consciousness: two equations with three unknowns. Prog Brain Res, 150, 427-444.

Kouider, S., \& Dehaene, S. (2007). Levels of processing during non-conscious perception: a critical review of visual masking. Philos Trans $R$ Soc Lond B Biol Sci, 362(1481), $857-875$.

Laureys, S. (2005). The neural correlate of (un)awareness: lessons from the vegetative state. Trends Cogn Sci, 9(12), 556-559.

Laureys, S., Celesia, G. G., Cohadon, F., Lavrijsen, J., Leon-Carrion, J., Sannita, W. G., et al. (2010). Unresponsive wakefulness syndrome: a new name for the vegetative state or apallic syndrome. BMC Med, 8, 68 .

Laureys, S., Owen, A. M., \& Schiff, N. D. (2004). Brain function in coma, vegetative state, and related disorders. Lancet Neurol, 3(9), 537-546.

Laureys, S., Pellas, F., Van Eeckhout, P., Ghorbel, S., Schnakers, C., Perrin, F., et al. (2005). The locked-in syndrome : what is it like to be conscious but paralyzed and voiceless? Prog Brain Res, 150, 495-511.

Luck, S. J. (2005). An Introduction to the Event-Related Potential Technique: MIT Press.

Manly, B. F. J. (1997). Randomization, Bootstrap and Monte Carlo Methods in Biology, Second Edition. Boca Raton, FL: Chapman \& Hall.

Monti, M. M., Vanhaudenhuyse, A., Coleman, M. R., Boly, M., Pickard, J. D., Tshibanda, L., et al. (2010). Willful modulation of brain activity in disorders of consciousness. $N$ Engl J Med, 362(7), 579-589.

Moruzzi, G., \& Magoun, H. W. (1949). Brain stem reticular formation and activation of the EEG. Electroencephalogr Clin Neurophysiol, 1(4), 455-473.

Mulckhuyse, M., \& Theeuwes, J. (2010). Unconscious attentional orienting to exogenous cues: A review of the literature. Acta Psychol (Amst), 134(3), 299-309.

Naatanen, R., Tervaniemi, M., Sussman, E., Paavilainen, P., \& Winkler, I. (2001). "Primitive intelligence" in the auditory cortex. Trends Neurosci., 24(5), 283-288.

Naccache, L. (2006). Psychology. Is she conscious? Science, 313(5792), 1395-1396.

Naccache, L. (2008). Visual consciousness : an updated neurological tour. In S. Laureys \& G. Tononi (Eds.), The neurology of consciouness : cognitive neuroscience and neuropathology (pp. 271-281). London: Academic Press.

Naccache, L., Gaillard, R., Adam, C., Hasboun, D., Clemenceau, S., Baulac, M., et al. (2005). A direct intracranial record of emotions evoked by subliminal words. Proc Natl Acad Sci U S A.

Naccache, L., Puybasset, L., Gaillard, R., Serve, E., \& Willer, J. C. (2005). Auditory mismatch negativity is a good predictor of awakening in comatose patients: a fast and reliable procedure. Clin Neurophysiol, 116(4), 988-989.

Niedermeyer, E. (2003). Electrophysiology of the frontal lobe. Clin Electroencephalogr, 34(1), 5-12.

Owen, A. M., Coleman, M. R., Boly, M., Davis, M. H., Laureys, S., \& Pickard, J. D. (2006). Detecting awareness in the vegetative state. Science, 313(5792), 1402. 
Owen, A. M., Coleman, M. R., Menon, D. K., Johnsrude, I. S., Rodd, J. M., Davis, M. H., et al. (2005). Residual auditory function in persistent vegetative state: a combined PET and fMRI study. Neuropsychol Rehabil, 15(3-4), 290-306.

Parvizi, J., \& Damasio, A. (2001). Consciousness and the brainstem. Cognition, 79(1-2), 135160.

Pegado, F., Bekinschtein, T., Chausson, N., Dehaene, S., Cohen, L., \& Naccache, L. Probing the lifetimes of auditory novelty detection processes. Neuropsychologia.

Plum, F., \& Posner, J. B. (1972). The diagnosis of stupor and coma. Contemp Neurol Ser, 10, 1-286.

Rama, P., Relander-Syrjanen, K., Ohman, J., Laakso, A., Naatanen, R., \& Kujala, T. (2010). Semantic processing in comatose patients with intact temporal lobes as reflected by the N400 event-related potential. Neurosci Lett, 474(2), 88-92.

Rockstroh, B., Elbert, T., Birbaumer, N., \& Lutzenberger, W. (1982). Slow brain potentials and behavior. Baltimore-Munich: Urban \& Schwarzenberg.

Rosahl, S. K., \& Knight, R. T. (1995). Role of prefrontal cortex in generation of the contingent negative variation. Cereb Cortex, 5(2), 123-134.

Schnakers, C., Majerus, S., Giacino, J., Vanhaudenhuyse, A., Bruno, M. A., Boly, M., et al. (2008). A French validation study of the Coma Recovery Scale-Revised (CRS-R). Brain Inj, 22(10), 786-792.

Schnakers, C., Perrin, F., Schabus, M., Hustinx, R., Majerus, S., Moonen, G., et al. (2009). Detecting consciousness in a total locked-in syndrome: an active event-related paradigm. NeuroCase, 15(4), 271-277.

Schnakers, C., Perrin, F., Schabus, M., Majerus, S., Ledoux, D., Damas, P., et al. (2008). Voluntary brain processing in disorders of consciousness. Neurology, 71(20), 16141620.

Schnakers, C., Vanhaudenhuyse, A., Giacino, J., Ventura, M., Boly, M., Majerus, S., et al. (2009). Diagnostic accuracy of the vegetative and minimally conscious state: clinical consensus versus standardized neurobehavioral assessment. BMC Neurol, 9, 35.

Sergent, C., Baillet, S., \& Dehaene, S. (2005). Timing of the brain events underlying access to consciousness during the attentional blink. Nat Neurosci, 8(10), 1391-1400.

Seth, A. K., Dienes, Z., Cleeremans, A., Overgaard, M., \& Pessoa, L. (2008). Measuring consciousness: relating behavioural and neurophysiological approaches. Trends Cogn Sci, 12(8), 314-321.

Thulasidas, M., Guan, C., \& Wu, J. (2006). Robust classification of EEG signal for braincomputer interface. IEEE Trans Neural Syst Rehabil Eng, 14(1), 24-29.

Tiitinen, H., May, P., Reinikainen, K., \& Naatanen, R. (1994). Attentive novelty detection in humans is governed by pre-attentive sensory memory. Nature, 372(6501), 90-92.

Velly, L. J., Rey, M. F., Bruder, N. J., Gouvitsos, F. A., Witjas, T., Regis, J. M., et al. (2007). Differential dynamic of action on cortical and subcortical structures of anesthetic agents during induction of anesthesia. Anesthesiology, 107(2), 202-212.

Walter, W. G., Cooper, R., Aldridge, V. J., McCallum, W. C., \& Winter, A. L. (1964). Contingent Negative Variation: an Electric Sign of Sensorimotor Association and Expectancy in the Human Brain. Nature, 203, 380-384.

Wijnen, V. J., van Boxtel, G. J., Eilander, H. J., \& de Gelder, B. (2007). Mismatch negativity predicts recovery from the vegetative state. Clin Neurophysiol, 118(3), 597-605. 


\section{Figures legends}

\section{Figure 1: Test design and illustration of bedside recording in intensive care-unit}

Top:

(a) On each trial five complex sounds of $50 \mathrm{~ms}$ duration each were presented with a fixed stimulus onset asynchrony of $150 \mathrm{~ms}$ between sounds. Four different types of series of sounds were used, the first two were prepared using the same five sounds (AAAAA or BBBBB), and the second two series of sounds were either AAAAB or BBBBA.

(b) Each block started with 20-30 frequent series of sounds to establish the global regularity before delivering the first infrequent global deviant stimulus.

Bottom:

Photography of a high-density EEG recording setting of a patient in intensive care-unit (with the authorization of patient family). Installation of the net and EEG calibration requires about 15 minutes. Earphones are then applied, task instruction delivered, and EEG recording starts.

\section{Figure 2: Local and global effects at the group level}

Left: Dynamics of Global Field Power (GFP) for the global regularity conditions (top panel), and for the local regularity conditions (bottom panel). Global and local standard (blue curves) and deviant (red curves) GFPs are plotted, and each significant difference between standard and deviant voltages is indicated by a black marker on the $\mathrm{X}$-axis (see M\&M for details).

Middle: Dynamics of ERPs on two regions of interests. Global effect is plotted around Pz (top panel), where a significant $\mathrm{P} 3 \mathrm{~b}$ component is observed exclusively for the conscious controls group. Local effect is plotted around $\mathrm{Fz}$ (bottom panel), where a large $\mathrm{MMN}$ is present for conscious controls, and decreases progressively in relation to the severity of consciousness impairment.

Right: ERP scalp topographies of the P3b global effect (top) averaged across the grey shadowed time-window (400-600 ms). Bottom topographies indicate the MMN local effect across the corresponding time-window (140-180 ms).

\section{Figure 3: Local effect at the individual level}

Each horizontal line corresponds to one ERP recording. On the left, clinical characteristics are indicated, including patient conscious state, and CRS-R scoring. Recordings are sorted from VS patients to conscious patients, and in each category from low to high CRS-R scores. For each recording, the presence of any significant ERP local effect, - tested with the triplethreshold t-test based statistics confirmed with Monte-Carlo permutation analyses detailed in M\&M -, is indicated by a color-code. A similar plotting is showed for each of the 8 controls where a MMN could be detected for each subject. The graph located at the bottom represents the mean of individual statistics for each group.

\section{Figure 4: Global effect at the individual level}

This figure was created using the very same design as in Figure 3. Note that a global effect could be detected in each of the 8 control subjects. In patients, global effects were observed only in conscious or MCS patients. The only 2 VS patients with a global effect showed clear behavioral signs of consciousness within the 3-4 days after recording, suggesting that they were conscious during ERP recording (see text). 


\section{Figure 5: CNV predicts global effect in patients}

\section{Top:}

In controls, scalp topography of voltage averaged from 0 to $600 \mathrm{~ms}$ after the onset of the first sound reveals an anterior negativity maximum around $\mathrm{Cz}$ and corresponding to an expectation CNV (Left).

Voltage averaged around $\mathrm{Cz}$ is plotted for each group of subjects, and linear regressions show a progressive decrease of CNV slope from controls to VS patients (Right).

\section{Middle:}

$\mathrm{CNV}$ slope is shown for each individual recording. Each recording was categorized as CNV+ (blue bars) or CNV- (red bars) after comparing individual distributions of single-trials slopes with zero.

\section{Bottom:}

Global (top) and local (bottom) dynamics of GFP are plotted separately for the CNV+ (left) and CNV- (right) patients groups. Global and local standard (blue curves) and deviant (red curves) GFPs are plotted, and each significant difference between standard and deviant voltages is indicated by a black marker on the X-axis. A global effect is observed in the $\mathrm{CNV}+$ group.

\section{Figure 6: Learning effect in controls}

Left: Scalp topography of test minus training ERPs of global standards trials averaged across 110-244 ms after first sound onset, in controls.

Right: Dynamics of Fz centered ERPs of training (red) and test (blue) averaged ERPs reveal two effects, one occurring before the fifth sound, and a second one occurring later and statistically weaker.

\section{SOM Figure S1: Scalp topographies of local and global effects}

Scalp topographies of local effects (top) and global effects (bottom) are shown for each group of patients and for conscious controls. For local effects, note the progressive improvement of MMN and P3a topographies across groups. For global effects, conscious controls showed a strong P3b effect. While no global effect could be observed in MCS and VS patients groups, conscious patients presented a clear sustained P3b topography with a much less intense voltage than controls.

\section{SOM Figure S2: Local and global effects at the group level (subset of 49 patients)}

Left: Dynamics of Global Field Power (GFP) for the global regularity conditions (top panel), and for the local regularity conditions (bottom panel). Global and local standard (blue curves) and deviant (red curves) GFPs are plotted, and each significant difference between standard and deviant voltages is indicated by a black marker on the $\mathrm{X}$-axis (see M\&M for details).

Middle: Dynamics of ERPs on two regions of interests. Global effect is plotted around Pz (top panel), where a significant $\mathrm{P} 3 \mathrm{~b}$ component is observed exclusively for the conscious controls group. Local effect is plotted around Fz (bottom panel), where a large MMN is present for conscious controls, and decreases progressively in relation to the severity of consciousness impairment. 
Right: ERP scalp topographies of the P3b global effect (top) averaged across the grey shadowed time-window (400-600 ms). Bottom topographies indicate the MMN local effect across the corresponding time-window (140-180 ms).

SOM Figure S3: CNV predicts global effect in patients (subset of 49 patients)

Global (top) and local (bottom) dynamics of GFP are plotted separately for the CNV+ (left) and CNV- (right) patients groups, within the restricted dataset of 49 patients with a single recording per patient. Global and local standard (blue curves) and deviant (red curves) GFPs are plotted, and each significant difference between standard and deviant voltages is indicated by a black marker on the X-axis. A global effect is observed in the $\mathrm{CNV}+$ group. 


\section{Acknowledgements}

This work has been supported by the Fondation pour la Recherche Médicale (FRM) (PhD support to Frédéric Faugeras and 'Equipe FRM 2010' grant to Lionel Naccache), by the JNLF (Master 2 funding to Frédéric Faugeras), by the ERC (NeuroConsc grant supporting Stanislas Dehaene and Lionel Naccache), by the Institut pour le Cerveau et la Moëlle épinière (ICM Institute, Paris, France), by INSERM and by AP-HP. We thank Pr. Chastre, Pr. Similowski, Pr. Samson, Pr. Rouby and Dr. Patte-Karsenti for addressing us some of the patients recorded in that study. This study is dedicated to the patients and to their close relatives. 